\title{
How has SPI changed in times of agile development? Results from a multi-method study
}

\author{
Steffen Küpper, Dietmar Pfahl, ${ }^{2}$ Kristjan Jürisoo, ${ }^{3}$ Philipp Diebold, Jürgen Münch, \\ Marco Kuhrmann ${ }^{6}$
}

\begin{abstract}
The emergence of agile methods and practices has not only changed the development processes but might also have affected how companies conduct software process improvement (SPI). Through a set of complementary studies, we aim to understand how SPI has changed in times of agile software development. Specifically, we aim (1) to identify and characterize the set of publications that connect elements of agility to SPI, (2) to explore to which extent agile methods/practices have been used in the context of SPI, and (3) to understand whether the topics addressed in the literature are relevant and useful for industry professionals. To study these questions, we conducted an in-depth analysis of the literature identified in a previous mapping study, an interview study, and an analysis of the responses given by industry professionals to SPI-related questions stemming from an independently conducted survey study.
\end{abstract}

This summary refers to the paper How has SPI changed in times of agile development? Results from a multi-method study [Kü19]. This paper was published as research article (empirical) in the Journal of Software: Evolution and Process.

Keywords: Agile Software Development; Software Process; Hybrid Development Approaches

\section{Introduction}

Software development processes have adopted many elements from agile methods and practices. That is, processes have become more agile and more hybrid. This brings up the question whether software process improvement (SPI) has become more agile as well. Do companies today still use standardized SPI frameworks like CMMI? Is SPI conducted in the form of projects with defined start and end dates, or is it a continuously performed activity that adapts to short-term goals and needs? Is SPI itself undergoing a change towards adopting an agile flavor, represented by short improvement cycles, adaptive improvement goals and flexible improvement actions?

\footnotetext{
${ }^{1}$ Clausthal University of Technology, Germany, steffen.kuepper@tu-clausthal.de

${ }^{2}$ University of Tartu, Estonia, dietmar.pfahl@ut.ee

${ }^{3}$ University of Tartu, Estonia, k.jyrisoo@gmail.com

${ }^{4}$ Bagilstein GmbH, Germany, kontakt@bagilstein.de

${ }^{5}$ Reutlingen University, Böblingen, Germany, Juergen.Muench@Reutlingen-University.de

${ }^{6}$ University of Passau, Germany, kuhrmann@acm.org
} 
Problem Statement \& Objective To answer the questions above, it is important to understand what experience exists regarding SPI in the context of agile and hybrid development processes. We aim to study the current state of the art and practice. We aim to better understand how agile methods/practices are used in the context of planning and conducting SPI.

Contribution The paper at hand presents contributes findings from a multitude-method research. Based on a systematic mapping study, we selected 55 publications for a systematic literature review. The complement the secondary studies, we conducted an expert interview with seven companies in Estonia and backed up the findings with data obtained in the first stage of the HELENA study [Ku17].

\section{Results}

We identified 55 publications that focus on both SPI and agility of which 48 present and discuss how agile methods/practices are used to steer SPI initiatives. We found that the two most frequently mentioned agile methods in the context of SPI are Scrum and Extreme Programming (XP), while the most frequently mentioned agile practices are integrate often, test-first, daily meeting, pair programming, retrospective, on-site customer, and product backlog. Furthermore, we found that a majority of the interviewed and surveyed industry professionals see SPI as a continuous activity. They agree with the agile SPI literature that agile methods and practices play an important role in SPI activities but that the importance given to specific agile methods and practices does not always coincide with the frequency with which these methods and practices are mentioned in the literature.

\section{Conclusion \& Future Work}

Our paper [Kü19] indicates that the trend towards agile SPI is visible both in the research literature and in surveys and interviews with industry professionals. Differences between these data sources exist regarding the prominence of specific agile development methods and practices in the context of SPI and the focus on implicit and continuous SPI versus explicit and strategic SPI.

\section{References}

[Ku17] Kuhrmann, Marco; Diebold, Philipp; Münch, Jürgen; Tell, Paolo; Garousi, Vahid; Felderer, Michael; Trektere, Kitija; McCaffery, Fergal; Linssen, Oliver; Hanser, Eckhart; Prause, Christian R.: Hybrid Software and System Development in Practice: Waterfall, Scrum, and Beyond. In: Proceedings of the 2017 International Conference on Software and System Process. ICSSP 2017, ACM, New York, NY, USA, pp. 30-39, 2017.

[Kü19] Küpper, Steffen; Pfahl, Dietmar; Jürisoo, Kristjan; Diebold, Philipp; Münch, Jürgen; Kuhrmann, Marco: How has SPI changed in times of agile development? Results from a multi-method study. Journal of Software: Evolution and Process, 31(11):1-28, 2019. 